\title{
Some Identities Associated with a Discriminant
}

\author{
By G. N. Watson
}

(Received 24th Noz ember 1953.)

\section{Introduction.}

This paper has to deal with some topics suggested by a theorem which is notorious in the theory of quadratic forms, namely that the roots of the characteristic equation of any real quadratic form are all real. For a proof of this theorem, the reader may consult Ferrar (1), p. 146.

Take the quadratic form to be one in three variables, say

$$
a x^{2}+b y^{2}+c z^{2}+2 u y z+2 v z x+2 w x y \text {, }
$$

with $a ; b, c, u, v, w$ all real, so that the characteristic equation is the cubic

$$
\left|\begin{array}{ccc}
a-\lambda & w & v \\
w & b-\lambda & u \\
v & u & c-\lambda
\end{array}\right|=0 .
$$

A change of the unknown from $\lambda$ to $\mu$ by the substitution

$$
3 \lambda=3 \mu+\Sigma a
$$

deprives the cubic of its second term; wo then write

$$
3 a^{\prime}=3 a-\Sigma a, \quad 3 b^{\prime}=3 b-\Sigma a, \quad 3 c^{\prime}=3 c-\Sigma a,
$$

and the characteristic equation becomes

wherein

$$
\left|\begin{array}{ccc}
a^{\prime}-\mu & w & v \\
w & b^{\prime}-\mu & u \\
v & u & c^{\prime}-\mu
\end{array}\right|=0
$$

This is of the form $\mu^{3}+3 H \mu+G=0$, with

$$
\begin{aligned}
& 9 H \equiv 3 \Sigma b^{\prime} c^{\prime}-3 \Sigma u^{2} \\
& \equiv \Sigma b c-\Sigma a^{2}-3 \Sigma u^{2} \\
& 2 \tau G \equiv-2 \succsim\left(a^{\prime} b^{\prime} c^{\prime}+2 u v w-\Sigma a^{\prime} u^{2}\right) \\
& \equiv-\Pi(2 a-b-c)-54 u v w+9 \Sigma(2 a-b-c) u^{2},
\end{aligned}
$$

and

the summations and product running over $a, b, c$, or over $u, v w$, or over $a, b, c$ and $u, v, w$ jointly. 
Now a necessary and sufficient condition for the reality of the three roots of a cubic equation with real coefficients is that the product of the squared differences of the roots should be positive in the loose sense. For the cubics in $\lambda$ and $\mu$ this product, is equal; to $-27\left(4 H^{3}+G^{2}\right)$; we thus obtain the following theorem, due to Kummer (2):

Theorem 1. Let

(1) $27 F(a, b, c ; u, v, w) \equiv 4\left(\Sigma a^{2}-\Sigma b c+3 \Sigma u^{2}\right)^{3}$,

then

$$
-\left\{\Pi(2 a-b-c)+54 u v w-9 \Sigma(2 a-b-c) u^{2}\right\}^{2} \text {; }
$$

$$
F(a, b, c ; u, v, w) \geqq 0
$$

for all real values of the numbers $a, b, c, u, v, w$.

It was remarked by Kummer that this theorem suggested that it might be possible to express $F(a, b, c ; u, v, v)$ as a sum of squares, and he proceeded to state such an expression after giving the corresponding formula for the special case $F(0,0,0 ; u, v, w)$; this expression is given by formula (14) infra. He gave no indication of how he discovered these results. Some forty ycars later Tannery (3) gave a derivation of the formulae by the methods of analytical geometry; although his work does not involve any complicated algebra, it seemed to me to be very obscure, and I decided that it might bo worth while to derive the formulae in as straightforward and natural a manner as possible; apart from quoting one theorem about invariance, I use nothing more than the elementary algebra of complex numbers.

It was noticed by Kummer that $a, b, c$ enter $F$ only by their differences; and we can abbreviate the subsequent work substantially by writing

$$
b-c=\alpha, \quad c-a=\beta, \quad a-b=\gamma,
$$

so that

$$
a+\beta+\gamma=0 .
$$

With this notation, the expansion of $F(a, b, c ; u, v, w)$ in ascending powers of $u, v, w$ assumes the form

$$
\begin{aligned}
& F(a, b, c ; u, v, w) \\
& \equiv \alpha^{2} \beta^{2} \gamma^{2}+\Sigma\left(4 \beta^{2} \gamma^{2}+2 a^{2} \beta \gamma\right) u^{2}+4 u v w \Pi(\beta-\gamma) \\
& +\Sigma\left(4 a^{2}+8 \beta \gamma\right) u^{4}+\Sigma\left(20 \alpha^{2}-2 \beta \gamma\right) v^{2} w^{2} \\
& -36 u v w \Sigma u^{2}(\beta-\gamma)+4\left(\Sigma u^{2}\right)^{3}-108 u^{2} v^{2} w^{2} .
\end{aligned}
$$

This result shows incidentally that, in the special case in which $a, b, c$ are all equal, Theorem 1 reduces to the theorem of the arithmetic and geometric means for $u^{2}, v^{2}, w^{2}$. 
2. Some special cases of the representation of $F(a, b, c ; u, v, w)$.

Before we attack the general problem of representing the expression $F(a, b, c ; u, v, w)$ as a sum of squares, we consider special cases in which at least one of the numbers $u, v, w$ is zero. First, when at least two of them are zero (say $u=v=0$ ), we have the almost trivial formula

$$
F(a, b, c ; 0,0, w) \equiv\left(\gamma^{2}+4 w^{2}\right)\left(\alpha \beta+w^{2}\right)^{2} .
$$

Next, let only one of the three numbers be zero (say $u=0, v \neq 0$, $w \neq 0$ ). We replace the variables $a, \beta, \gamma$ by new variables $a_{1}, \beta_{1}, \gamma_{1}$ defined by the formulæ

$$
a_{1}=\frac{a}{\sqrt{ }\left(v^{2}+w^{2}\right)}, \quad \beta_{1}=\frac{\beta}{\sqrt{ }\left(v^{2}+w^{2}\right)}, \quad \gamma_{1}=\frac{\gamma}{\sqrt{ }\left(v^{2}+w^{2}\right)} .
$$

This change transforms $F(a, b, c ; 0, v, w) /\left(v^{2}+w^{2}\right)$ into a homogencous quadratic function of $v^{2}$ and $w^{2}$, the coefficients in the quadratic being functions of $a_{1}, \beta_{1}, \gamma_{1}$. In fact, some straightforward simplifications show that

$$
\begin{aligned}
& F(a, b, c ; 0, v, w) /\left(v^{2}+w^{2}\right) \\
& \equiv v^{4}\left(\beta_{1}^{2}+4\right)\left(\alpha_{1} \gamma_{1}+1\right)^{2}+w^{4}\left(\gamma_{1}{ }^{2}+4\right)\left(\alpha_{1} \beta_{1}+1\right)^{2} \\
& \quad+v^{2} w^{2}\left\{2 \alpha_{1}{ }^{2}{\beta_{1}}^{2}{\gamma_{1}}^{2}+{a_{1}}^{2}\left(4{\beta_{1}}^{2}+4{\gamma_{1}}^{2}-2 \beta_{1} \gamma_{1}\right)\right. \\
& \left.+\left(19 \alpha_{1}{ }^{2}+{\beta_{1}}^{2}+\gamma_{1}{ }^{2}\right)+8\right\}
\end{aligned}
$$

An inspection of the expression on the right shows that the whole. of the terms in $v^{4}$ and $w^{4}$, together with a great part of the term in $v^{2} w^{2}$, is accounted for by the sum

$$
\left\{v^{2} \beta_{1}\left(a_{1} \gamma_{1}+1\right)+w^{2} \gamma_{1}\left(a_{1} \beta_{1}+1\right)\right\}^{2}+4\left\{v^{2}\left(a_{1} \gamma_{1}+1\right)+w^{2}\left(a_{1} \beta_{1}+1\right)\right\}^{2} ;
$$

in fact, when wo subtract this sum from the expression in question and use (4) freely to reduce the result, we are left with

$$
v^{2} w^{2}\left\{4 a_{1}^{2}\left(\beta_{1}-\gamma_{1}\right)^{2}+27 a_{1}^{2}+\left(\beta_{1}-\gamma_{1}\right)^{2}\right\},
$$

and, by good fortune, this is a sum of squares. Hence we have

$$
\begin{aligned}
F( & (a, b, c ; 0, v, w) /\left(v^{2}+w^{2}\right) \\
\equiv & \left\{\left(v^{2}+w^{2}\right) a_{1} \beta_{1} \gamma_{1}+v^{2} \beta_{1}+w^{2} \gamma_{1}\right\}^{2} \\
& +4\left\{v^{2} a_{1} \gamma_{1}+w^{2} a_{1} \beta_{1}+v^{2}+w^{2}\right\}^{2} \\
& +4 v^{2} w^{2} a_{1}^{2}\left(\beta_{1}-\gamma_{1}\right)^{2}+v^{2} w^{2}\left\{27 a_{1}^{2}+\left(\beta_{1}-\gamma_{1}\right)^{2}\right\} .
\end{aligned}
$$


When we return to the original notation, this result becomes

$$
\begin{aligned}
& F(a, b, c ; 0, v, w) \cdot\left(v^{2}+w^{2}\right) \\
& \equiv\left(v^{2}+w^{2}\right)\left(a \beta \gamma+v^{2} \beta+w^{2} \gamma\right)^{2} \\
& +4\left\{v^{2} a \gamma+w^{2} \alpha \beta+\left(v^{2}+w^{2}\right)^{2}\right\}^{2}+4 v^{2} w^{2} \alpha^{2}(\beta-\gamma)^{2} \\
& \quad+v^{2} w^{2}\left(v^{2}+w^{2}\right)\left\{2 \alpha^{2}+(\beta-\gamma)^{2}\right\} .
\end{aligned}
$$

This result can be simplificd by rearranging the second and third terms on tha right in such a way that it becomes possible to cancel a factor $v^{2}+w^{2}$. To effect the rearrangement, observe that

$$
\begin{aligned}
v^{2} a \gamma+w^{2} a \beta & +\left(v^{2}+w^{2}\right)^{2} \pm i v w a(\beta-\gamma) \\
& \equiv(v \mp i w)\left\{v\left(\alpha \gamma+v^{2}+w^{2}\right) \pm i w\left(\alpha \beta+v^{2}+w^{2}\right)\right\},
\end{aligned}
$$

where either all the upper signs or else all the lower signs are to be taken throughout the ambiguities. By means of this pair of formulæ we derive the following theorem from (i).

Theorem 2. The special function $F(a, b, c ; 0, v, w)$ can be repre. sented as a sum of squares by the formula

$$
\begin{aligned}
F(a, b, c ; 0, v, w) \equiv(\alpha \beta \gamma+ & \left.v^{2} \beta+w^{2} \gamma\right)^{2} \\
& +4 v^{2}\left(\alpha \gamma+v^{2}+w^{2}\right)^{2} \\
& +4 w^{2}\left(\alpha \beta+v^{2}+w^{2}\right)^{2} \\
& +v^{2} w^{2}\left\{27 a^{2}+(\beta-\gamma)^{2}\right\} .
\end{aligned}
$$

It was assumed that $v^{2}+v^{2}$ is not zero in the course of proving Theorem 2, but it is evident that the theorem remains valid when this limitation is removed.

3. Representations of the general function $F(a, b, c ; u, v, w)$.

Now that we have disposed of various special cases, we direct our attention to the general function $F(a, b, c ; u, v, w)$ in which it is not assumed that $u v w$ is zcro. We derive identities from Theorem 2 by a process which is quite straightforward but rather tedious.

It is well known (Ferrar, 1, p. 194) that the coefficients in the characteristic equation of a quadratic form are invariant under normal orthogonal transformations of the form; we take a special transformation of this type, namely

$$
x=X, \quad y=Y \cos \theta-Z \sin \theta, \quad z=Y \sin \theta+Z \cos \theta,
$$

with a value of $\theta$ which will be chosen presently; the original form

$$
a x^{2}+b y^{2}+c z^{2}+2 u y z+2 v z x+2 w x y
$$

is thereby converted into (say) 
with

$$
A X^{2}+B Y^{2}+C Z^{2}+2 U Y Z+2 V Z X+2 W X Y,
$$

$$
\left\{\begin{array}{l}
A=a, \quad U=\frac{1}{2}(c-b) \sin 2 \theta+u \cos 2 \theta, \\
B=b \cos ^{2} \theta+c \sin ^{2} \theta+2 u \sin \theta \cos \theta, \\
C=b \sin ^{2} \theta+c \cos ^{2} \theta-2 u \sin \theta \cos \theta, \\
V=v \cos \theta-w \sin \theta, W=v \sin \theta+w \cos \theta .
\end{array}\right.
$$

Since the product of the squared differences of the roots of a cubic equation is a rational function of the coeffisients in the equation, the theorem on invariance which has just been quoted shows that

$$
F(a, b, c ; u, v, w) \equiv F(A, B, C ; U, V, W) ;
$$

this result, of course, can be proved by a lengthy picce of elementary algebra, as a dircet consequence of equations (9), without any appeal to theorems on invariance.

We now choose $\theta$ so that $U$ vanishes, that is to say wo tako

$$
\cos 2 \theta=\frac{a}{K}, \quad \sin 2 \theta=\frac{2 u}{K},
$$

where $R$ denotes $\pm \sqrt{ }\left(a^{2}+4 u^{2}\right)$; it does not matter whether the positive or the negative value is assigned to $R$ so long as one value is adopted throughout the work. With a value of $\theta$ satisfying (11) wo have

$$
\begin{aligned}
& F(a, b, c ; u, v, w) \equiv F(A, B, C ; 0, V, W) \\
& \equiv\left\{(B-C)(C-A)(A-B)+V^{2}(C-A)+W^{2}(A-B)\right\}^{2} \\
& \quad+4 V^{2}\left\{(B-C)(A-B)+V^{2}+W^{2}\right\}^{2} \\
& \quad+4 W^{2}\left\{(B-C)(C-A)+V^{2}+W^{2}\right\}^{2} \\
& \quad+V^{2} W^{2}(B+C-2 A)^{2}+27 V^{2} W^{2}(B-C)^{2} .
\end{aligned}
$$

We now have to express the right-hand side of (12) as a sum of squares of functions of $a, \beta, \gamma, u, v, w$. As a preliminary we give the following results, which are easily obtained from (9):

$$
\begin{gathered}
B-C=a \cos 2 \theta+2 u \sin 2 \theta=R, \\
B+C-2 A=\beta-\gamma, \\
V W=v w \cos 2 \theta+\frac{1}{2}\left(v^{2}-w^{2}\right) \sin 2 \theta \\
=R^{-1}\left\{v w a+u\left(v^{2}-w^{2}\right)\right\}, \\
4(C-A)(A-B)=(B-C)^{2}-(B+C-2 A)^{2} \\
=4 \beta \gamma+4 u^{2}, \\
2(V \pm i W)\{V(C-A) \pm i W(B-A)\} \\
=(V \pm i W)^{2}(B+C-2 A)-\left(V^{2}+W^{2}\right)(B-C) \\
=e^{ \pm 2 i \theta}(v \pm i w)^{2}(B-\gamma)-R\left(v^{2}+w^{2}\right) .
\end{gathered}
$$


Of the five terms on the right of (12), the fifth gives no troublo since it is evidently equal to $27\left\{v w a+u\left(v^{2}-w^{2}\right)\right\}^{2}$. We deal with the first and fourth terms by expressing their sum as the product of conjugate complex factors and observing that

$$
\begin{aligned}
(B-C) & (C-A)(A-B)+V^{2}(C-A)+W^{2}(A-B) \pm i V W(B+C-2 A) \\
& =R\left(\beta \gamma+u^{2}\right)+(V \pm i W)\{V(C-A) \pm i W(B-A)\} \\
& =R\left(\beta \gamma+u^{2}-\frac{1}{2} v^{2}-\frac{1}{2} w^{2}\right)+\frac{1}{2} e^{ \pm 2 i \theta}(v \pm i w)^{2}(\beta-\gamma) \\
& =e^{ \pm 2 i \theta}\left\{(a \mp 2 i u)\left(\beta \gamma+u^{2}-\frac{1}{2} v^{2}-\frac{1}{2} w^{2}\right)+\frac{1}{2}(v \pm i w)^{2}(\beta-\gamma)\right\} \\
& =e^{ \pm 2 i \theta}\left[a \beta \gamma+\Sigma a u^{2} \mp i\left\{2 u \beta \gamma-v w(\beta-\gamma)+u\left(2 u^{2}-v^{2}-w^{2}\right)\right\}\right] .
\end{aligned}
$$

We deal with the sum of the second and third terms on the right of (12) in a similar manner by observing that

$$
\begin{aligned}
& 2 V\left\{(B-C)(A-B)+V^{2}+W^{2}\right\} \pm 2 i W\left\{(B-C)(C-A)+V^{2}+W^{2}\right\} \\
& =(V \pm i W)\left\{2 V^{2}+2 W^{2}-(B-C)^{2}\right\}-(V \mp i W)(B-C)(B+C-2 A) \\
& =e^{ \pm i \theta}(v \pm i w)\left(2 v^{2}+2 w^{2}-a^{2}-4 u^{2}\right)-e^{\mp i \theta}(v \mp i w) R(\beta-\gamma) \\
& =e^{ \pm i \theta}\left\{(v \pm i w)\left(2 v^{2}+2 w^{2}-a^{2}-4 u^{2}\right)-(v \mp i w)(a \mp 2 i u)(\beta-\gamma)\right\} \\
& =2 e^{ \pm i \theta}\left[\left\{v a \gamma+u w(\beta-\gamma)+v\left(v^{2}+w^{2}-2 u^{2}\right)\right\}\right. \\
& \left.\quad \pm i\left\{w a \beta+u v(\beta-\gamma)+w\left(v^{2}+w^{2}-2 u^{2}\right)\right\}\right] .
\end{aligned}
$$

When wo substitute these results in (12), we obtain an identity which is curiously unsymmetrical in appearance; it is comprised in the following theorem.

Theorem 3. The general function $F(a, b, c ; u, v, w)$ can be represented as a sum of squares by the formula

$$
\begin{aligned}
F(a, b, c ; u, v, w) & \equiv\left(\alpha \beta \gamma+\Sigma a u^{2}\right)^{2} \\
+ & 4\left\{v \alpha \gamma+u w(\beta-\gamma)+v\left(v^{2}+w^{2}-2 u^{2}\right)\right\}^{2} \\
+ & 4\left\{w a \beta+u v(\beta-\gamma)+w\left(v^{3}+w^{2}-2 u^{2}\right)\right\}^{2} \\
+ & \left\{2 u \beta \gamma-v w(\beta-\gamma)+u\left(2 u^{2}-v^{2}-w^{2}\right)\right\}^{2} \\
+ & 27\left\{v w \alpha+u\left(v^{2}-w^{2}\right)\right\}^{2} .
\end{aligned}
$$

This identity appears to be new. To deduco from it an identity which is symmetrical in appearance (but of greater length when written in full), change $a, b, c$ and $u, v, w$ cyclically and sum. This process gives

$$
\begin{aligned}
3 F(a, b, c ; u, v, w) \equiv & 3\left(a \beta \gamma+\Sigma a u^{2}\right)^{2} \\
& +4 \Sigma\left\{u \beta \gamma+v w(a-\beta)+u\left(u^{2}+v^{2}-2 w^{2}\right)\right\}^{2} \\
& +4 \Sigma\left\{u \beta \gamma+v w(\gamma-\alpha)+u\left(w^{2}+u^{2}-2 v^{2}\right)\right\}^{2} \\
& +\Sigma\left\{2 u \beta \gamma-v w(\beta-\gamma)+u\left(2 u^{2}-v^{2}-w^{2}\right)\right\}^{2} \\
& +27 \Sigma\left\{v w a+u\left(v^{2}-u^{2}\right)\right\}^{2} .
\end{aligned}
$$


Now apply the formula

$$
4 p^{2}+4 q^{2}=2(p+q)^{2}+2(p-q)^{2}
$$

to like terms of the sums in the second and third lines of this result. Wo immediately obtain Kummer's theorem as follows:

Theorem 4. The general function $F(a, b, c ; u, v, w)$ can be represented as a sum of squares by the formula

$$
\begin{aligned}
F(a, b, c ; u, v, w) & \equiv\left(a \beta \gamma+\Sigma \alpha u^{2}\right)^{2} \\
& +\Sigma\left\{2 u \beta \gamma-v w(\beta-\gamma)+u\left(2 u^{2}-v^{2}-w^{2}\right)\right\}^{2} \\
& +15 \Sigma\left\{v w \alpha+u\left(v_{-}^{2}-w^{2}\right)\right\}^{2} .
\end{aligned}
$$

If we take $a=b=c$ in (14) and compare the result with (5), we obtain an unusual proof of the theorem of the arithmetic and geometric means for three variables.

Since this special case $a=b=c$ is the only special case of (14) which is mentioned by Kummer, it seems highly probable that his method of constructing (14) and the method which I have just given are completely different.

The reader may find it an interesting example to obtain the ordinary conditions for the characteristic equation to have a pair of equal roots directly from (14).

\section{REFERENCES.}

1. W. L. Ferrar, Algebrn (Oxford, 1940).

2. E. E. Kummer, Journul für Math., 26 (1843), 268-272.

3. J. Tannery, Bulletin des sci. math. et astr. (2), 7 (1883), 103-107.

46 WaRWICK NeW ROAD, Leaminaton, WarWickshiRe. 